\title{
Ultraviolet and Optical Emission Line Outflows in the Heavily Obscured Quasar SDSS J000610.67+121501.2: At the Scale of the Dusty Torus and Beyond
}

\author{
Shaohua Zhang ${ }^{1}$, Hongyan Zhou ${ }^{1,2}$, Xiheng Shi ${ }^{1}$, Xiang Pan ${ }^{1,2}$, Ji Wang ${ }^{3}$, Ning Jiang ${ }^{2}$, Tuo $\mathrm{Ji}^{1}$, Peng Jiang ${ }^{1}$, Wenjuan Liu ${ }^{4}$, and \\ Huiyuan Wang $^{2}$ \\ ${ }^{1}$ Polar Research Institute of China, 451 Jinqiao Road, Shanghai, 200136, China; zhangshaohua@ pric.org.cn, zhouhongyan@pric.org.cn \\ ${ }^{2}$ Key Laboratory for Research in Galaxies and Cosmology, Department of Astronomy, University of Sciences and Technology of China, \\ Chinese Academy of Sciences, Hefei, Anhui 230026, China \\ ${ }^{3}$ California Institute of Technology, 1200 East California Boulevard, Pasadena, CA 91101, USA \\ ${ }^{4}$ Yunnan Observatories, Chinese Academy of Sciences, Kunming, Yunnan 650011, China \\ Received 2016 August 26; revised 2017 January 15; accepted 2017 January 17; published 2017 February 10
}

\begin{abstract}
Broad emission line outflows of active galactic nuclei have been proposed for many years but are very difficult to quantitatively study because of the coexistence of the gravitationally bound and outflow emission. We present detailed analysis of a heavily reddened quasar, SDSS J000610.67+121501.2, whose normal ultraviolet broad emission lines (BELs) are heavily suppressed by the dusty torus as a natural "coronagraph," and thus the blueshifted BELs (BBELs) can be reliably measured. The physical properties of the emission-line outflows are derived as follows: ionization parameter $U \sim 10^{-0.5}$, column density $N_{\mathrm{H}} \sim 10^{22.0} \mathrm{~cm}^{-2}$, covering fraction of $\sim 0.1$, and upper limit density of $n_{\mathrm{H}} \sim 10^{5.8} \mathrm{~cm}^{-3}$. The outflow gases are located at least $41 \mathrm{pc}$ away from the central engine, which suggests that they have expanded to the scale of the dust torus or beyond. Besides, Ly $\alpha$ shows a narrow symmetric component, to our surprise, which is undetected in any other lines. After inspecting the narrow emission line region and the star-forming region as the origin of the Ly $\alpha$ narrow line, we propose that the end result of outflows, diffusing gases in the larger region, acts as the screen of Ly $\alpha$ photons. Future high spatial resolution spectrometry and/or spectropolarimetric observations are needed to make a final clarification.
\end{abstract}

Key words: galaxies: active - galaxies: individual (SDSS J000610.67+121501.2) - galaxies: interactions quasars: emission lines

\section{Introduction}

The blueshifted emission lines (blueshifted-ELs) and broad absorption lines (BALs), which are the typical representations of active galactic nucleus (AGN) outflows, have been widely studied. They are potentially one of the most important pieces of feedback from AGNs and may play a crucial role in galaxy formation and evolution, connecting the central supermassive black holes (SMBHs) to their host galaxies and regulating their coevolution (e.g., Granato et al. 2004; Scannapieco \& Oh 2004; Hopkins et al. 2008). Previous works suggested that the AGN outflows can reach from the small scale (at or outside of the broad emission line region [BELR]; e.g., Zhang et al. 2015c; Williams et al. 2016) to the intermediate scale (the dusty torus; e.g., Leighly et al. 2015; Zhang et al. 2015b; Shi et al. 2016), the large scale (the inner narrow emission line region [NELR]; e.g., Komossa et al. 2008; Crenshaw et al. 2010; Ji et al. 2015; Liu et al. 2016), and even the entire host galaxy of the quasar (e.g., Zakamska et al. 2016). There is general agreement that there has to be a radial component of motion and some opacity source; however, questions regarding the origins, whether homologous or not, and the properties themselves of the blueshifted-ELs and BALs remain interesting subjects of debate. They can also be used as important test beds for models of the BELR, broad absorption line region, and NELR.

Comparatively, studies on the blueshifted-ELs are more difficult than those on the BALs. This is because the BALs have high-speed blueshifted (up to a velocity of $v \sim 0.2 c$ ) and broad-width (at least $2000 \mathrm{~km} \mathrm{~s}^{-1}$ ) troughs from a wide range of species, such as Mg II, Al III, Fe II, N v, C IV, Si IV, and O vi (e.g., Weymann et al. 1991; Hall et al. 2002; Reichard et al. 2003; Trump et al. 2006; Gibson et al. 2010; Zhang et al.
2010), and even from the hydrogen Balmer series (e.g., Hall 2007; Ji et al. 2012, 2013; Zhang et al. 2015b) and metastable He I (e.g., Liu et al. 2015, 2016), whose obvious features allow the BALs to be easily detected and measured. Unfortunately, the broad and narrow blueshifted-ELs (BBELs and BNELs) are generally mixed with the normal broad or narrow lines. The existence of the blueshifted-ELs is expressed by the differences in the line profile (e.g., asymmetry, width, peak, and/or velocity centroid) with respect to the lines emitting from the BELR and NELR (e.g., Gaskell 1982; Wilkes 1984; Crenshaw 1986; Marziani et al. 1996, 2013; Richards et al. 2002; Zamanov et al. 2002; Boroson 2005; Snedden \& Gaskell 2007; Crenshaw et al. 2010; Rafiee et al. 2016), and the strength measurement relies on the blueshift and asymmetry index for C IV (Wang et al. 2011, 2013), or the distinguishing of the blueshifted wings for [O III] $\lambda 5007$ (e.g., Komossa et al. 2008; Zhang et al. 2011; Zakamska et al. 2016) and for $\mathrm{H} \beta$ and $\mathrm{Mg}$ II (e.g., Marziani et al. 2013).

Though the fundamental parameters of AGNs that affect the blueshifted-ELs have been found, such as the Eddington ratio (e.g., Komossa et al. 2008; Wang et al. 2011), the physical properties and locations of these outflows are hard to investigate, except for a limited number of sources, in which the spectra of the blueshifted-ELs from a range of species can be reliably measured. For example, Leighly \& Moore (2004) identified broad, strongly blueshifted high-ionization lines of $\mathrm{CIV}$ and $\mathrm{NV}$, which are dominated by emission from the wind in two extreme narrow-line Seyfert 1 galaxies $\left(\mathrm{FWHM}_{\mathrm{MgII}} \lesssim 1000 \mathrm{~km} \mathrm{~s}^{-1}\right)$ : IRAS $13224-$ 3809 and $1 \mathrm{H}$ 0707-495. The photoionization analysis favors a wide range of densities $\left(\log n_{\mathrm{H}} \sim 7-11 \mathrm{~cm}^{-3}\right)$ and a relatively high ionization parameter $(\log U \sim-1.2$ to -0.2$)$. Moreover, the 
Table 1

Photometric Observations of SDSS J0006+1215

\begin{tabular}{lcllc}
\hline \hline Band & Magnitude & Date of Observation & Facility & Reference \\
\hline$u$ & $24.53 \pm 1.13$ & 2008 Nov. 02 & SDSS & 1 \\
$g$ & $22.13 \pm 0.09$ & 2008 Nov. 02 & SDSS & 1 \\
$r$ & $22.06 \pm 0.13$ & 2008 Nov. 02 & SDSS & 1 \\
$i$ & $22.11 \pm 0.18$ & 2008 Nov. 02 & SDSS & 1 \\
$z$ & $20.70 \pm 0.23$ & 2008 Nov. 02 & SDSS & 1 \\
$Y$ & $20.33 \pm 0.28$ & 2010 Jul. 26 & UKIDSS & 2 \\
$J$ & $18.97 \pm 0.11$ & 2010 Jul. 26 & UKIDSS & 2 \\
$H$ & $17.37 \pm 0.05$ & 2010 Jul. 26 & UKIDSS & 2 \\
$K$ & $15.81 \pm 0.03$ & 2010 Jul. 26 & UKIDSS & 2 \\
$W 1$ & $14.32 \pm 0.03$ & 2010 Jun. 28 & WISE & 3 \\
$W 2$ & $12.74 \pm 0.03$ & 2010 Jun. 28 & WISE & 3 \\
$W 3$ & $8.85 \pm 0.03$ & 2010 Jun. 28 & WISE & 3 \\
$W 4$ & $6.55 \pm 0.07$ & 2010 Jun. 28 & WISE & 3 \\
\hline
\end{tabular}

References. (1) Ahn et al. (2014); (2) Lawrence et al. (2007); (3) Wright et al. (2010).

blueshifted-EL emission regions and the BELRs do not differ in the location, as $\sim 10^{4} r_{S}$ was inferred for both (Leighly 2004). Liu et al. (2016) identified the above three phenomena produced by the AGN outflows in the optical to near-infrared (NIR) spectra of SDSS J163459.82+204936.0, i.e., a cuspy BNEL component in $\mathrm{H} \beta$, He I $\lambda \lambda 5876,10830$, a BBEL component of He I $\lambda 10830$, the bulk blueshifting of [O III] $\lambda 5007$, and BALs in Na I D and He I $\lambda \lambda 5876,10830$. The physical parameters determined with Cloudy for absorption-line and emission-line outflows are very close, with $\log n_{\mathrm{H}} \sim 4.5-5 \mathrm{~cm}^{-3}$ and $\log U \sim-1.3$ to -1.0 , and the outflow materials are 48-65 pc from the central exterior of the torus. Of special interest is the similarity of the physical parameters and the locations, which strongly suggest that blueshifted-ELs and BALs should be generated in the common outflowing gas.

In a study of the intermediate-width emission lines (IELs), Li et al. (2015) used the dusty torus as a "coronagraph," which heavily suppresses the normal broad emission lines (BELs) in O I 287; thus, the ultraviolet (UV) IELs can be reliably detected from the quasar emission line spectra. This work reminds us that partially obscured quasars may provide an opportunity to reliably detect the blueshifted-ELs. If the blueshifted-ELs do exist, they would become prominent in the shorter wavelength range where the normal BELs are heavily suppressed. In this work, we report such an infrared-luminous quasar, J000610.67 +121501.2 (hereafter SDSS J0006+1215), at $z=2.3174$ with outflows revealed in $\mathrm{CIV}, \mathrm{Ly} \alpha$, and $\mathrm{NV}$ BBELs. The organization of this paper is as follows. The data we used are described in Section 2. We fit the spectrum and analyze the UV BBELs in Section 3, and we discuss the properties and possible origins of BBEL outflows in Section 4. A summary of our results is given in Section 5. Throughout this paper, we adopt the cold dark matter "concordance" cosmology with $H_{0}=70 \mathrm{~km} \mathrm{~s}^{-1} \mathrm{Mpc}^{-1}, \Omega_{\mathrm{m}}=0.3$, and $\Omega_{\Lambda}=0.7$.

\section{Observations}

SDSS J0006 +1215 is an infrared-luminous quasar with faint UV emission. It was first imaged by the Sloan Digital Sky Survey (SDSS; York et al. 2000) at the five optical wide bands. Meanwhile, the UKIRT Infrared Deep Sky Surveys (UKIDSS; Lawrence et al. 2007) and the Wide-field Infrared Survey Explorer (WISE; Wright et al. 2010) provide the NIR

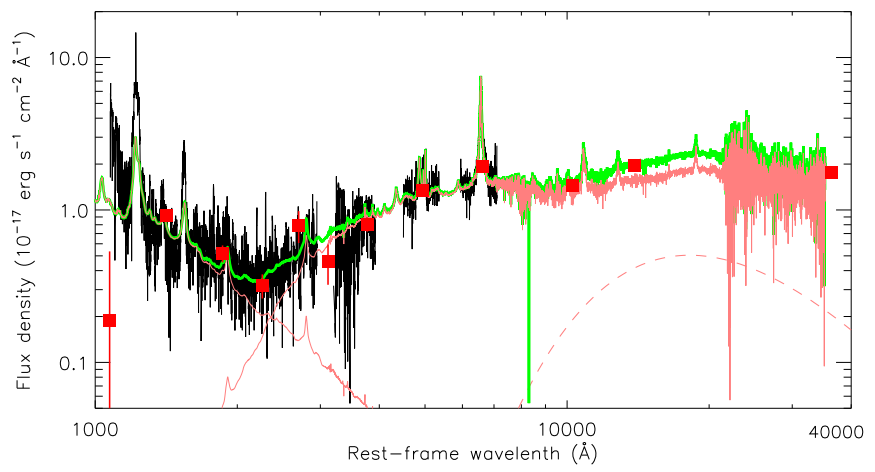

Figure 1. Broadband SED of SDSS J0006+1215 from UV to MIR (red squares) and the spectra of SDSS and TripleSpec (black curves). The scattering light, the scaled and reddened quasar composite, the hot dust emission, and their sum are shown by pink and green curves.

to mid-infrared (MIR) photometric data for SDSS J0006 +1215 . The photometric data are summarized in Table 1.

The optical spectrum of SDSS J0006+1215 was taken with the SDSS $2.5 \mathrm{~m}$ telescope on 2011 December 16, in the SDSSIII Baryon Oscillation Spectroscopic Survey (BOSS; Dawson et al. 2013), and published in the SDSS Tenth Data Release (DR10; Ahn et al. 2014). The BOSS spectrum has a wider wavelength range, covering 361-1014 $\mathrm{nm}$ with a resolution of 1300 at the blue side and 2600 at the red side. This instrument is fed by smaller optical fibers, each subtending $2^{\prime \prime}$ on the sky (Smee et al. 2013).

The NIR spectrum of SDSS J0006+1215 was performed with the TripleSpec spectrograph of the Hale 200-inch telescope (P200) at Palomar Observatory on 2015 December 4. Four exposures of $240 \mathrm{~s}$ each are taken in an A-B-B-A dithering model. TripleSpec (Wilson et al. 2004) provides simultaneous wavelength coverage from 0.9 to $2.46 \mu \mathrm{m}$ at a resolution of 1.4-2.9 A, with two gaps at approximately 1.35 and $1.85 \mu \mathrm{m}$ owing to the telluric absorption bands. Fortunately, the redshifted $\mathrm{H} \beta$ and $\mathrm{H} \alpha$ emission lines are detected with TripleSpec at $J$ and $K_{s}$ band, respectively. The raw data were processed using Interactive Data Language (IDL) based Spextool software (Vacca et al. 2003; Cushing et al. 2004).

\section{Data Analysis and Results}

\subsection{Broadband Spectral Energy Distribution}

After correcting for the Galactic reddening of $E(B-V)=$ $0.078 \mathrm{mag}$ (Schlegel et al. 1998), we transformed the photometric data and the optical and NIR spectroscopic flux into the rest-frame values with an emission redshift of $z=2.3174 \pm 0.0003$, which is carefully determined by fitting a Gaussian to the Ly $\alpha$ narrow line. The photometric and spectroscopic data are shown by red squares and black and blue curves in Figure 1. Note that the absolute flux calibration is operated based on the SDSS and UKIDSS photometry for both optical and NIR spectra. With the multiwavelength spectroscopic and photometric data, we construct the broadband spectral energy distribution (SED) of SDSS J0006+1215 spanning from $1100 \AA$ to $6.65 \mu \mathrm{m}$ in the rest frame. For comparison, the quasar composite is composed of the SDSS DR7 quasar composite $(\lambda \leqslant 3000 \AA$; Jiang et al. 2011) and the NIR template $(\lambda>3000 \AA$; Glikman et al. 2006). It is obvious that the observed SED of SDSS J0006 +1215 shows a very different shape from the quasar composite. There is a striking " $\mathrm{V}$ "-shape 
turning at $i$ band in the SED of SDSS J0006+1215, which is likely to be caused by a type of excess broadband absorption or the spectral combination of two different continuum slopes.

For the first case, the unusual shape is quite similar to the excess broadband absorption near $2250 \AA$ (EBBA) in BAL quasars reported by Zhang et al. (2015a). Therefore, the spectrum. of SDSS J0006+1215 is fit to search the quasarassociated $2175 \AA$ absorber using the quasar composite reddened by a parameterized extinction curve (Fitzpatrick \& Massa 1990) in the quasar rest frame, where a Drude component represents the potential $2175 \mathrm{~A}$ bump (more details can be found in Section 2 of Zhang et al. 2015a). For SDSS J0006+1215, the parameters of the Drude component are $c 3=92.07 \pm 3.83$, $x_{0}=4.71 \pm 0.02 \mu \mathrm{m}^{-1}$, and $\gamma=5.32 \pm 0.26 \mu \mathrm{m}^{-1}$. The bump strength is $A_{\text {bump }}=\pi c 3 /(2 \gamma)=27.16 \pm 1.76 \mu \mathrm{m}$. Apparently, the width $(\gamma)$ and strength $\left(A_{\text {bump }}\right)$ of the bump deviate heavily from those of the bumps for the Milky Way (Fitzpatrick \& Massa 1990), LMC (Gordon et al. 2003) curves, and the quasar-associated $2175 \AA$ extinction curves (Jiang et al. 2011; Zhang et al. 2015a) (see Figure 2 of Zhang et al. 2015a). Therefore, the broadband absorption model is not preferred.

For the second case, the fibers feeding the SDSS-III BOSS spectrograph subtend a diameter of $2^{\prime \prime}$ on the sky, and light from quasar pairs with small angular separations can be included through such a fixed aperture. The physical size, to which the diameter corresponds, indicates that the spectrum of SDSS J0006 +1215 would also be polluted by its host galaxy. However, the Ly $\alpha$ and $\mathrm{H} \alpha$ emission lines simultaneously presented in the blue and red ends of the optical-NIR spectrum suggest that both spectral components of SDSS J0006+1215 could be quasar-type spectra. Thus, we try the combination of a red quasar spectrum and a blue one, which are the dominant components of the spectrum of SDSS J0006+1215 with $3000 \AA \lesssim \lambda \lesssim 7000 \AA$ and $\lambda \lesssim 2000 \AA$, respectively. Here, we use (1) a quasar composite scaled and multiplied by the SMC extinction law with a free parameter $E(B-V)$ to refer to the red component, and (2) a quasar composite scaled by a power-law form $\left(\propto \lambda^{-\alpha_{\lambda}}\right)$ to match the warped blueward. In addition, a hot dust emission component is used to add the emission lacking in the infrared composite $(\lambda \gtrsim 9000 \AA)$. Finally, we can decompose the broadband SED in rest-frame wavelength with the following model:

$$
\begin{aligned}
F_{\lambda}= & C_{1} \lambda^{-\alpha_{\lambda}} F_{\text {composite }, \lambda}+C_{2} A \\
& \times(E(B-V), \lambda) F_{\text {composite }, \lambda}+C_{3} B_{\lambda}\left(T_{\text {dust }}\right),
\end{aligned}
$$

where $C_{1}, C_{2}$, and $C_{3}$ are the factors for the respective components, $A(E(B-V), \lambda)$ is the dust extinction to the quasar emission, $F_{\text {composite }, \lambda}$ is the quasar composite, and $B_{\lambda}\left(T_{\text {dust }}\right)$ is the Planck function. We perform least-squares minimization using the IDL procedure MPFIT developed by $\mathrm{C}$. Markwardt. ${ }^{5}$ The values of $E(B-V)$ and $\alpha_{\lambda}$ are $0.7512 \pm$ 0.0049 and $1.1388 \pm 0.0061$, respectively. The typical temperature of hot dust is $T \sim 1600 \mathrm{~K}$. In Figure 1, the sum and three components of the best-fit model are shown by green and pink curves.

The fitting results suggest that the continuum of SDSS J0006 +1215 is heavily suppressed by dust reddening (see also, Ross et al. 2015; Hamann et al. 2017); however, the warped

\footnotetext{
5 The Markwardt IDL Library is available at http://cow.physics.wisc.edu/ $\sim$ craigm/idl/idl.html
}

blueward spectrum is even bluer than the average slope of quasars. We notice that the broadband SED of SDSS J0006 +1215 is similar to those of three high polarized quasars, i.e., PKS 2355-535 (Scarpa \& Falomo 1997), O I 287 (Li et al. 2015), and SDSS J091501.71+241812.1. ${ }^{6}$ This provides a guess that the warped blueward spectrum of SDSS J0006 +1215 is the scattered component arising from an extensive region. In such a situation, the warped blueward spectrum would present high polarized fluxes; unfortunately, the faint magnitudes of SDSS J0006+1215 are unfit for the current spectropolarimetry observation.

\subsection{Emission-line Spectrum}

The spectrum of SDSS J0006+1215 shows strong Ly $\alpha$ $+\mathrm{N}$ V, Si IV $+[\mathrm{O}$ IV $], \mathrm{C}$ IV, $\mathrm{H} \beta+[\mathrm{O} \mathrm{III}]$, and $\mathrm{H} \alpha$ emission lines; however, ambiguous [C III $]+[\mathrm{Si}$ III $]+\mathrm{Al}$ III and $\mathrm{Mg}$ II emission lines are submerged in large noises. To further study the emission lines, we first subtract the underlying pseudocontinuum from the observed spectra. In this work, linear functions $\left(f_{\lambda}=c_{1} * \lambda+c_{2}\right)$ estimated from two continuum windows are used as the local continua of the emission lines. In $\mathrm{H} \beta$ $+[\mathrm{O}$ III $]$ and $\mathrm{H} \alpha$ regimes, we also adopt the I Zw 1 Fe II template provided by Véron-Cetty et al. (2004) and convolve it with a Gaussian kernel in velocity space to match the width of Fe II multiplets in the observed spectra. The continuum windows are $[1160,1180] \AA$ and $[1270,1290] \AA$ for Ly $\alpha$ $+\mathrm{N} \mathrm{V},[1320,1350] \AA$ and $[1430,1450] \AA$ for Si IV + [O IV], $[1480,1500] \AA$ and $[1600,1620] \AA$ for C IV,$[4320,4720] \AA$ and [5080, 5300] $\AA$ for $\mathrm{H} \beta+[\mathrm{O}$ III], and [6150, 6250] $\AA$ and $[6850,6950] \AA$ for $\mathrm{H} \alpha$. In the right panels of Figure 2, the local continua and Fe II multiplets are marked by gray dashed and pink lines. After subtracting the continuum model, we show the profiles of emission lines in their common velocity space in the left panels of Figure 2.

It is also clearly shown that the peaks of broad lines in SDSS J0006+1215 tend to be blueshifted with respect to the Ly $\alpha$ narrow line, which indicates that the profiles of these broad lines contain an emission-line outflow component. In particular, the line profiles of $\mathrm{C} \mathrm{IV}$ and $\mathrm{Ly} \alpha+\mathrm{N} \mathrm{V}$ of SDSS J0006 +1215 are almost the same as those of IRAS 13224-3809 and 1H 0707-495 reported by Leighly \& Moore (2004). The profile of the C IV line is even entirely blueshifted (top left panel); the unusual $\mathrm{C} \mathrm{IV} / \mathrm{H} \alpha$ width ratio, $\sim 0.71$, is larger than the value in the quasar composite spectrum (see Table 2). Those suggest that the normal UV BELs of SDSS J0006+1215 might also be obscured, as is the continuum. If this is true, the CIV profile we observed is dominated by the emission-line outflows and the gravitationally bound component, i.e., the normal BEL, of C IV is possibly absent. Here, we start the line profile fittings with the C IV line by one Gaussian. The peak velocity of the CIV BBEL is blueshifted $2119 \mathrm{~km} \mathrm{~s}^{-1}$, and the FWHM is $4821 \mathrm{~km} \mathrm{~s}^{-1}$. Later, we use the modeled profile of the C IV line to recover the BBEL outflows of other emission lines.

First, the emission line of Si IV is depicted by the scaled C IV profile. Actually, the strength of the Si IV line is polluted by the emission of [O IV] $\lambda \lambda 1397,1399,1401,1404,1407$ (see Figure 7

\footnotetext{
6 SDSS J0915+2418 was observed with the TripleSpec spectrograph of the Hale 200-inch telescope (P200) at Palomar Observatory on 2014 January 18 and the SPOL CCD imaging/spectropolarimeter at the $6.5 \mathrm{~m}$ MMT on 2016 April 3. The MMT/SPOL observation shows that the mean polarized degree of the optical spectrum is $\sim 15 \%$ (C.-W. Yang, et al. 2016, in preparation).
} 

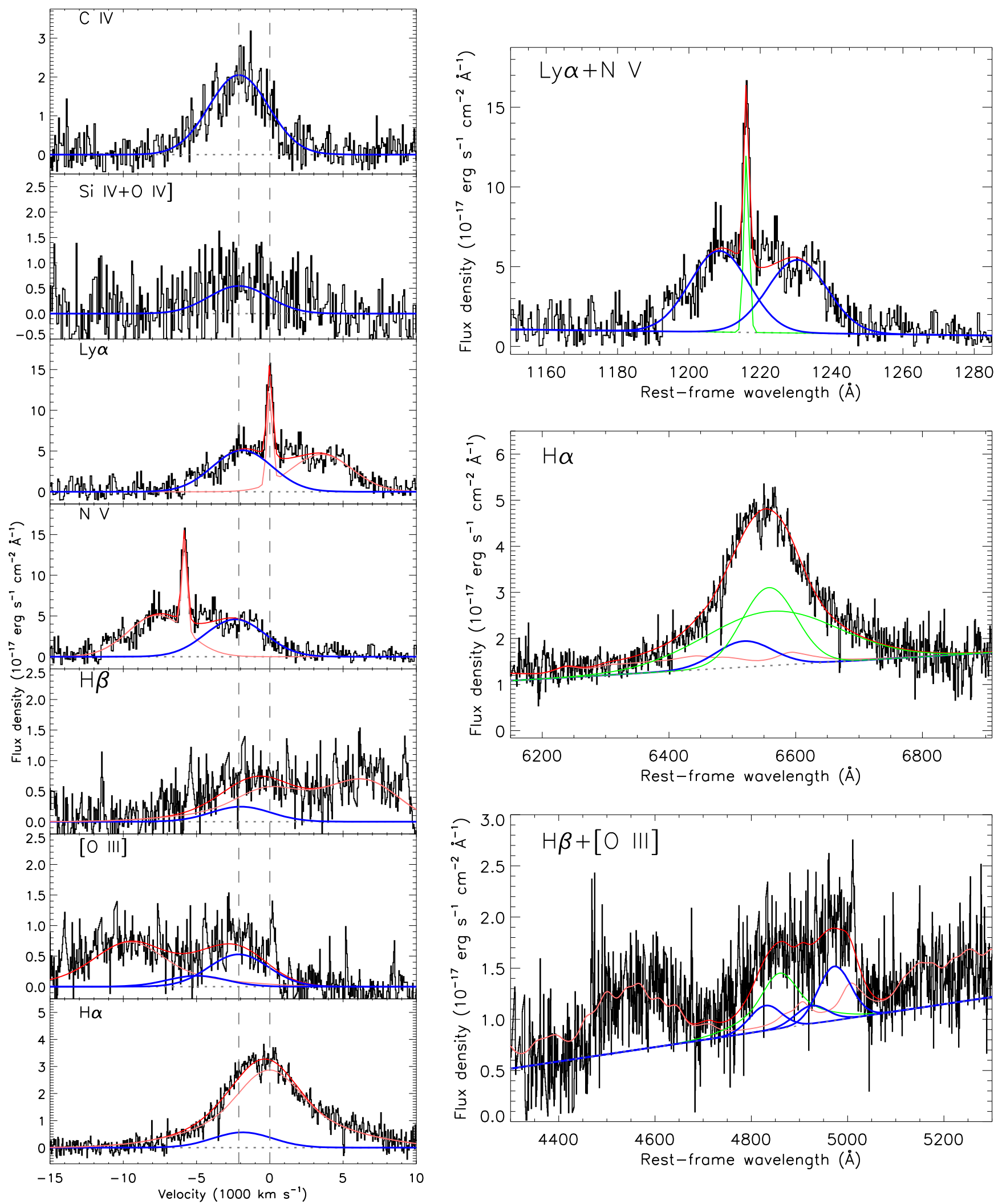

Figure 2. Left panels: demonstration of the profile of the various emission lines, particularly the blueshifted components caused by outflows. Plotted are the observed spectra with continuum subtracted (black lines), the total fitted profile of each emission line (red lines), the sum of the unblueshifted components of the corresponding line and other lines (only in the $\mathrm{Ly} \alpha+\mathrm{N} \mathrm{V}$ or $\mathrm{H} \beta+[\mathrm{O} \mathrm{III}]$ regimes) (pink lines), and the broad blueshifted component (blue lines). The gray dashed vertical lines indicate $-2119 \mathrm{~km} \mathrm{~s}^{-1}$ and $0 \mathrm{~km} \mathrm{~s}^{-1}$. Right panels: best-fit models for the Ly $\alpha+\mathrm{N} \mathrm{v}, \mathrm{H} \alpha$, and $\mathrm{H} \beta+[\mathrm{O}$ III] regions. The red lines represent the total fit profile for each region. The blue lines represent the blueshifted components, the green lines show the decomposed Gaussian profiles of the broad and narrow emission lines, respectively, the broadened optical Fe II template is in pink, and the gray dashed lines represent the local continua. 
Table 2

Emission-line Parameters of SDSS J0006+1215

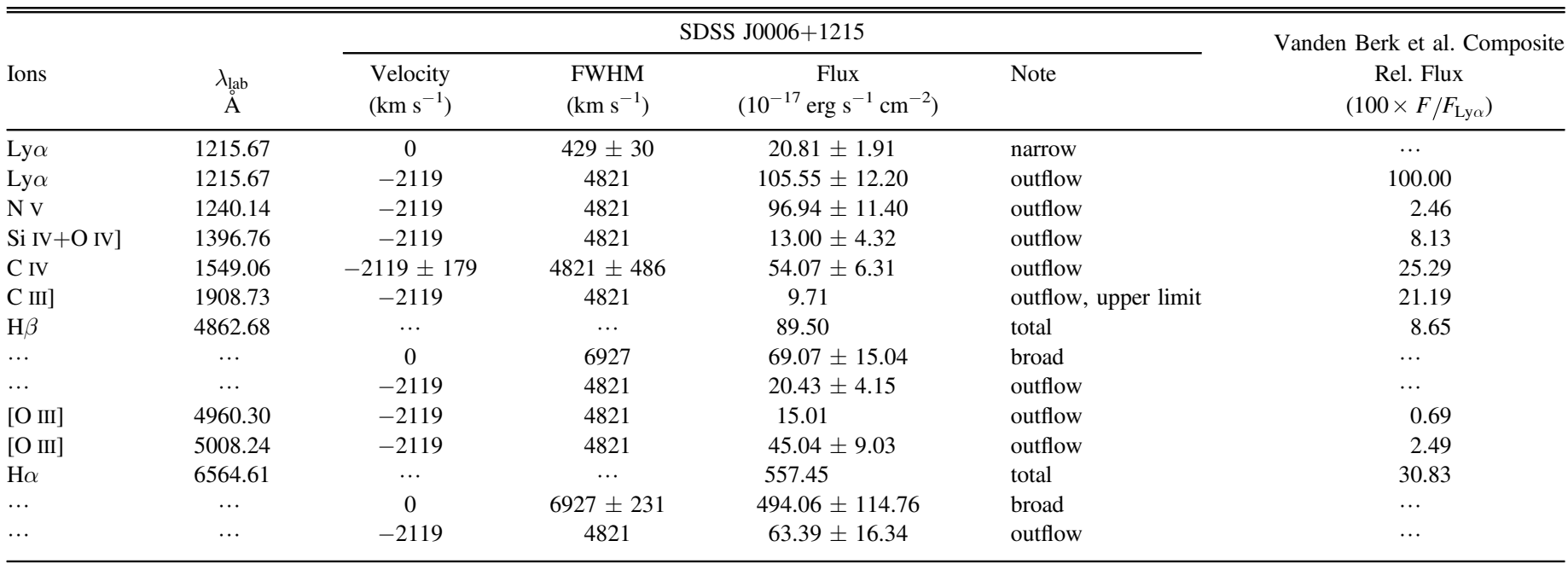

of Vanden Berk et al. 2001). A similar approach is taken in the fitting of the Ly $\alpha$ and $\mathrm{NV}$ lines, where the normal BEL components are absent. Then we use one narrow Gaussian component to model the $\operatorname{Ly} \alpha$ narrow line and two broad Gaussian components to model the Ly $\alpha$ and Nv BBEL components. The shifts and widths of broad Gaussian components are tied to those of the C IV line. In the top right panel, the sum of the local continuum (gray dotted line) and the Ly $\alpha$ narrow Gaussian (green line) plus the Ly $\alpha$ and N V BBEL outflows (blue lines) can well recover the observed spectrum.

Compared with UV emission lines, hydrogen Balmer lines in the optical waveband are still dominated by the gravitationally bound BELRs, and their profiles might only contain weak BBEL outflows. To separate the hydrogen Balmer emission line outflows, we use three Gaussian profiles to model the entire profile of the $\mathrm{H} \alpha$ line: one, with the shift and width settings of the C IV line, represents the BBEL outflows, and the other two, with the center wavelengths set to $6564.41 \AA$, represent the normal $\mathrm{H} \alpha$ BEL. They are shown by blue and green lines in the middle right panel, respectively. In the $\mathrm{H} \beta$ $+[\mathrm{O}$ III $]$ regime, we use the unshifted two-Gaussian profile of the $\mathrm{H} \alpha$ line to model the normal $\mathrm{H} \beta$ BEL (green line) and three Gaussians (blue lines) with the same shifts and widths as the $\mathrm{C}$ IV line to model the BBEL outflows of the $\mathrm{H} \beta$ and [O III] doublets in sequence. The details of these components are shown in the bottom right panel of Figure 2.

For all of the emission lines, we list the best-fit parameters in Table 2. Here, let us revert to the left panels of Figure 2. We show the total fitted profile of each emission line by red lines and the sum of the unblueshifted components of the corresponding line (and other lines only in the Ly $\alpha+\mathrm{N} \mathrm{V}$ or $\mathrm{H} \beta+[\mathrm{O}$ III] regimes) by pink lines. The differences between the two above items are the BBEL outflows of each line (blue line). The fit between the observed spectra with the continuumsubtracted and the total fitted profiles indicates the rationality of the existence of the BBEL outflows in all emission lines. Although the shifts of the peaks of the $\mathrm{H} \alpha$ line and the profile fitting of the $\mathrm{H} \beta+[\mathrm{O} \mathrm{III}]$ regime strongly suggest the existence of hydrogen Balmer line outflows, it must be said that the BBEL strengths of hydrogen Balmer lines have large measurement uncertainties because of the multicomponent decomposition.

The errors of line fluxes provided by MPFIT do not account for the uncertainty introduced by the pseudocontinuum subtraction. To take this and other possible effects into account, we adopt a bootstrap approach to estimate the typical errors for our emission-line measurement. We generate 500 spectra by randomly combining the scaled model outflow emission lines (denoted as "A") and the scaled model unblueshifted emission lines (denoted as "B") with the scaled model pseudocontinuum (denoted as "C"). Then, we fit the simulated spectra following the same procedure as described in the above paragraphs. For each parameter, we consider the typical error to be the standard deviation of the relative difference between the input $\left(x_{i}\right)$ and the recovered $\left(x_{o}\right)$, $\left(x_{o}-x_{i}\right) / x_{i}$. These relative differences turn out to be normally distributed for every parameter. Thus, the estimated typical $1 \sigma$ errors are $\gtrsim 10 \%$ for $\mathrm{C} I V, \mathrm{Ly} \alpha$, and $\mathrm{N} \mathrm{V}$ and $\gtrsim 20 \%$ for $\mathrm{H} \alpha, \mathrm{H} \beta$, and [O III]; the derived flux errors are listed in Table 2.

Various interesting facts are summarized as follows: (1) The Ly $\alpha$ narrow line is the only narrow emission line in the spectrum of SDSS J0006+1215. The FWHM of the Ly $\alpha$ narrow line is $429 \pm 30 \mathrm{~km} \mathrm{~s}^{-1}$, and the flux is $(20.81 \pm 0.89) \times 10^{-17} \mathrm{erg} \mathrm{s}^{-1}$. (2) The extreme C IV line with $\mathrm{EW}_{\mathrm{C} \text { IV }}=117.79 \pm 8.10 \AA$ far exceeds that of typical quasars $\left(\mathrm{EW}_{\mathrm{C} \text { IV }} \approx 20-50 \AA\right)$. SDSS J0006+1215 and 12 other extremely red objects with $\mathrm{EW}_{\mathrm{C} \text { IV }} \geqslant 100 \AA$ are actually classified as Extreme EW objects, which were considered to possibly be caused by suppressed continuum emission analogous to type II quasars in the unified model in Ross et al. (2015). However, flux emitted from the abnormal BELR is the other important reason, and it is interpreted as the BBEL outflows in this work. (3) The $\mathrm{H} \alpha / \mathrm{H} \beta$ flux ratio of their unblueshifted components is $7.15 \pm 2.27$; however, the intrinsic value of $\mathrm{H} \alpha / \mathrm{H} \beta$ of the BELR is 3.06, with a standard deviation of 0.03 dex (Dong et al. 2008). The extremely high flux ratio suggests that the extinction of the BELR is $E(B-V)=0.92_{-0.34}^{+0.26}$, which does not contradict the extinction we measured from the continuum of SDSS J0006+1215. (4) However, the $\mathrm{H} \alpha / \mathrm{H} \beta$ flux ratio of the outflowing 
components is $3.10 \pm 1.01$, which suggests that the outflow emission region is not obscured and reddened.

\section{Discussion}

\subsection{Central Black Hole and Underrated Luminosity}

From the emission-line fittings, we can derive the central black hole mass using the commonly used virial mass estimators. We use the broad $\mathrm{H} \alpha$ line-based mass formalism given by Greene \& Ho (2005):

$$
\begin{aligned}
M_{\mathrm{BH}}= & \left(2.0_{-0.3}^{+0.4}\right) \times 10^{6}\left(\frac{L_{\mathrm{H} \alpha}}{10^{42} \mathrm{erg} \mathrm{s}^{-1}}\right)^{0.55 \pm 0.02} \\
& \times\left(\frac{\mathrm{FWHM}_{\mathrm{H} \alpha}}{10^{3} \mathrm{~km} \mathrm{~s}^{-1}}\right)^{2.06 \pm 0.06} M_{\odot}
\end{aligned}
$$

where $L_{\mathrm{H} \alpha}$ and $\mathrm{FWHM}_{\mathrm{H} \alpha}$ are the luminosity and width of the $\mathrm{H} \alpha$ line, respectively. Here, we only use the unblueshifted component emitted from the gravitationally bound BELR. Supposing that the BELs also follow the continuum extinction, the corrected luminosity of the normal $\mathrm{H} \alpha$ BEL is $L_{\mathrm{H} \alpha}=4.74 \times 10^{45} \mathrm{erg} \mathrm{s}^{-1}$. Together with $\mathrm{FWHM}_{\mathrm{H} \alpha}=$ $6927 \mathrm{~km} \mathrm{~s}^{-1}$, the central black hole mass is estimated to be $M_{\mathrm{BH}}=1.13 \times 10^{10} M_{\odot}$, with an uncertainty of a factor of $\sim 2.6$ (from the intrinsic scatter of $\sim 0.41$ dex for this singleepoch method compared with the results of reverberation mapping; Ho \& Kim 2015). The monochromatic continuum luminosity $L_{5100}=\lambda L_{\lambda}$ at $5100 \AA$ is directly calculated from the local continuum of the $\mathrm{H} \beta$ regime, and the extinctioncorrected luminosity $L_{5100}=6.46 \times 10^{46} \mathrm{erg} \mathrm{s}^{-1}$. The bolometric luminosity, $L_{\mathrm{bol}}=2.30 \times 10^{47} \mathrm{erg} \mathrm{s}^{-1}$, is estimated from the monochromatic luminosity, $L_{5100}$, using the conversion given by Runnoe et al. (2012). Our bootstrap approach showed that the typical $1 \sigma$ error of the continuum under $\mathrm{H} \beta$ $+[\mathrm{O}$ III $]$ emission lines is $\sim 10 \%$, and thus the uncertainty of $L_{\mathrm{bol}}$ is also on the order of $10 \%$. The derived Eddington ratio is thus $l_{\mathrm{E}}=L_{\mathrm{bol}} / L_{\mathrm{Edd}}=0.17$. Based on the bolometric luminosity, the amount of mass being accreted is estimated as $\dot{M}_{\mathrm{acc}}=L_{\mathrm{bol}} / \eta c^{2}=41 M_{\odot} \mathrm{yr}^{-1}$, where we assumed an accretion efficiency $\eta$ of 0.1 , and $c$ is the speed of light.

From the point of view of UV-band photometric and spectroscopic observations, SDSS J0006+1215 is a fairly blue but faint quasar. The relative color and absolute magnitude at $i$ band are $\Delta(g-i)=-0.22$ and $M_{i}=-24.17$, bluer and fainter than most quasars in the SDSS-III/BOSS. If our inference regarding the unusual SED of SDSS J0006+1215 is true, it is heavily obscured with $E(B-V)=0.75$. And the above-mentioned characters of color and luminosity are measured based on the scattering component. The luminosity of SDSS J0006+1215 is heavily underestimated; when we compare the UV magnitudes and spectrum of SDSS J0006 +1215 with the quasar composite, it is easy to see that the observed magnitudes of SDSS J0006+1215 are fainter than the representation by approximately $4.5 \mathrm{mag}$. Following the quasar luminosity functions measured in the SDSS-III/ BOSS and SDSS-IV/eBOSS (e.g., Ross et al. 2013; Palanque-Delabrouille et al. 2016), we can derive the quasar number density with similar redshift and luminosity as follows: $\varnothing\left(z \sim 2.2-2.6, M_{i} \sim-28\right) \sim 10^{-8} \mathrm{Mpc}^{-3} \mathrm{mag}^{-1}$. The total spectroscopic footprint of the BOSS DR12 is approximately $10,400 \mathrm{deg}^{2}$, and the value without masked regions due to bright stars and data that do not meet the survey requirement is $9376 \mathrm{deg}^{2}$. If there are quite a number $(\sim 100)$ of quasars that have an SED and redshift similar to those of SDSS J0006+1215, that will redouble the bright end of the quasar luminosity function and lead to the statistical aberrations regarding the type I and type II ratio of the luminous AGNs at an intermediate redshift. A statistical sample study in the future would be very useful to sort out this question.

\subsection{Physical Properties of the Outflows}

As described in Section 3.2, the decomposition of the emission-line profiles indicates the presence of outflows in emission as revealed by the blueshifted components of C IV, Ly $\alpha, \mathrm{N}$ v, [O III] $\lambda \lambda 4959,5007$, and hydrogen Balmer lines, with a similar symmetric profile with a blueshifted velocity of $2119 \mathrm{~km} \mathrm{~s}^{-1}$ and an FWHM of $4821 \mathrm{~km} \mathrm{~s}^{-1}$. In this subsection, we analyze and determine the physical properties of the emission-line outflows using the photoionization synthesis code Cloudy (the latest version, last described by Ferland et al. 1998).

To investigate the physical properties for the emission-line outflows, we use the Cloudy simulations and confront these models with the measured line ratios to determine the density $\left(n_{\mathrm{H}}\right)$, column density $\left(N_{\mathrm{H}}\right)$, and ionization parameter $(U)$. We know that the BBEL manifests itself well in C IV, Si IV+[O IV], Ly $\alpha$, and $\mathrm{NV}$, with the entire emission-line profile being blueshifted since the normal BELs are heavily suppressed, and the BBEL also dominates the emission of [O III]. However, the $\mathrm{Si}$ IV+[O IV] BBEL is weak, with a large uncertainty. Moreover, the resonant scattering can contribute a significant part of the $\mathrm{N} \mathrm{V}$ line, and it may give rise to an anomalous strong $\mathrm{N} \mathrm{V}$ line (Hamann et al. 1993; Wang et al. 2007). Even in some objects, e.g., 0105-265, almost half of the Ly $\alpha$ fluxes are scattered (Ogle et al. 1999). Thus, Ly $\alpha, \mathrm{C}$ IV, and [O III] are chosen to confront the models, and Si IV $+[\mathrm{O}$ IV] and the absent [C III] are just used to examine the reasonability of the constrained parameter space. We consider that the BBEL emission region is not obscured, and adjacent lines are not urged. It is better for us to use the line ratios with large differences in ionization potential, i.e., [O III] $\lambda 55007 / \mathrm{Ly} \alpha$ and $\mathrm{C}$ IV/Ly $\alpha$, in order to probe different zones in a gas cloud. Furthermore, the critical density $\left(n_{\text {crit }}\right)$ of [O III] $\lambda \lambda 4959,5007$ is $7.0 \times 10^{5} \mathrm{~cm}^{-3}$, and we thus infer that the BBEL outflows should originate from the less dense part of the outflows, with a density lower than $n_{\text {crit }}$.

We consider a gas slab illuminated by a continuum source in the extensive parameter space. The outflowing gases are assumed to have uniform density and a homogeneous chemical composition of solar values and be free of dust. The incident SED applied is a typical AGN multicomponent continuum described as a combination of a blackbody "Big Bump" and power laws. ${ }^{7}$ The "Big Bump" component peaks at $\approx 1$ Ryd and is parameterized by $T=1.5 \times 10^{5} \mathrm{~K}$. The slope of the X-ray component, the X-rayto-UV ratio, and the low-energy slope are set to be $\alpha_{\mathrm{x}}=-1$, $\alpha_{\mathrm{ox}}=-1.4$, and $\alpha_{\mathrm{UV}}=-0.5$, respectively. This UV-soft SED is regarded as more realistic for radio-quiet quasars than the other available SEDs provided by Cloudy (see the detailed discussion in

\footnotetext{
7 See details in Hazy, a brief introduction to Cloudy; http://www. nublado.org.
} 


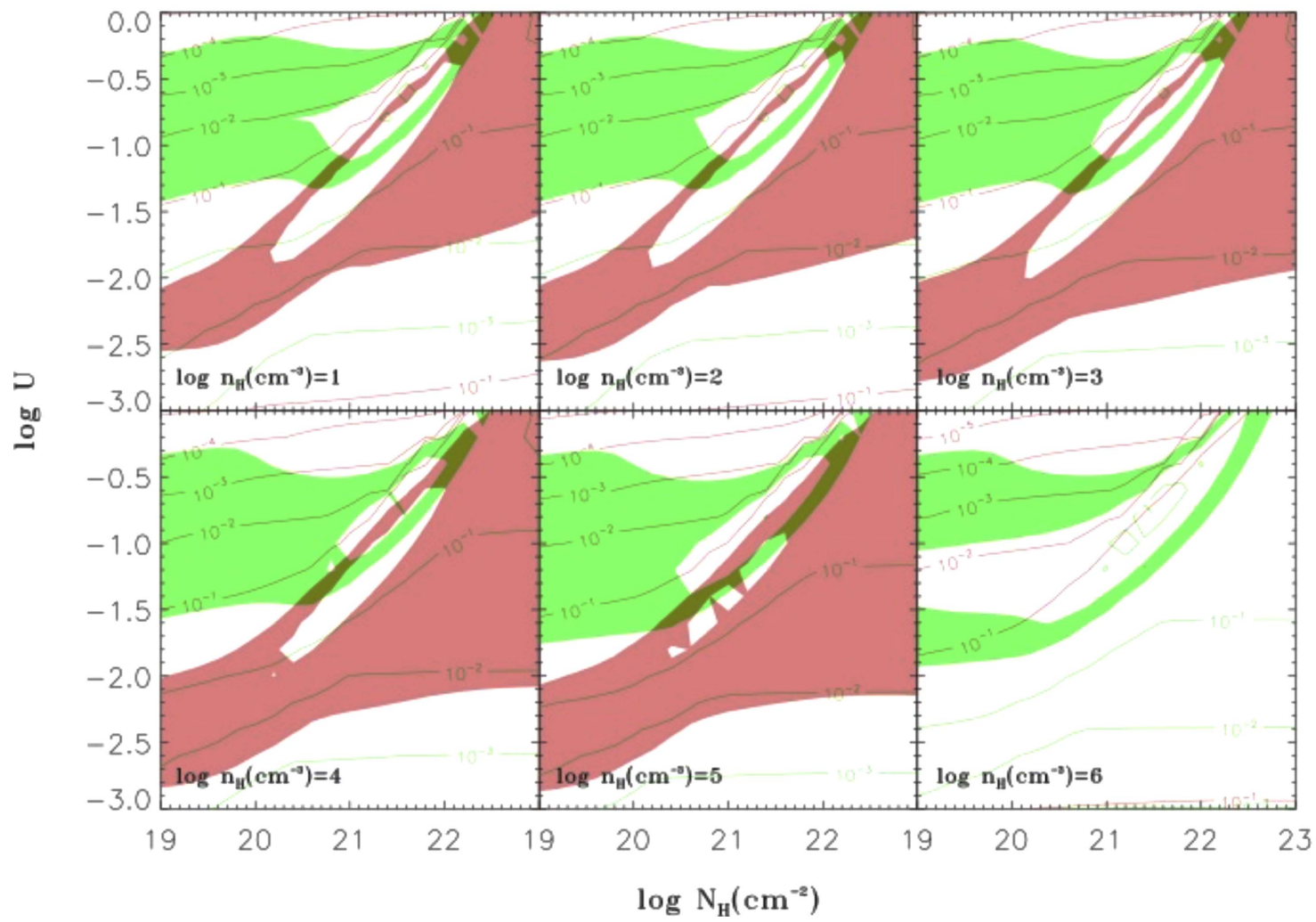

Figure 3. Photoionization models of the emission-line outflows in SDSS J0006+1215 assuming solar abundances. Red and green dashed lines represent the contours of flux ratios of [O III] $\lambda 5007 / \mathrm{Ly} \alpha$ and $\mathrm{C}$ IV $/ \mathrm{Ly} \alpha$, respectively. The red and green areas show the observed $1 \sigma$ uncertainty ranges of [O III] $\lambda 5007 / \mathrm{Ly} \alpha$ and $\mathrm{C}$ IV/Ly $\alpha$; the overlapping region is the possible parameter space for the BBEL outflows of this object.

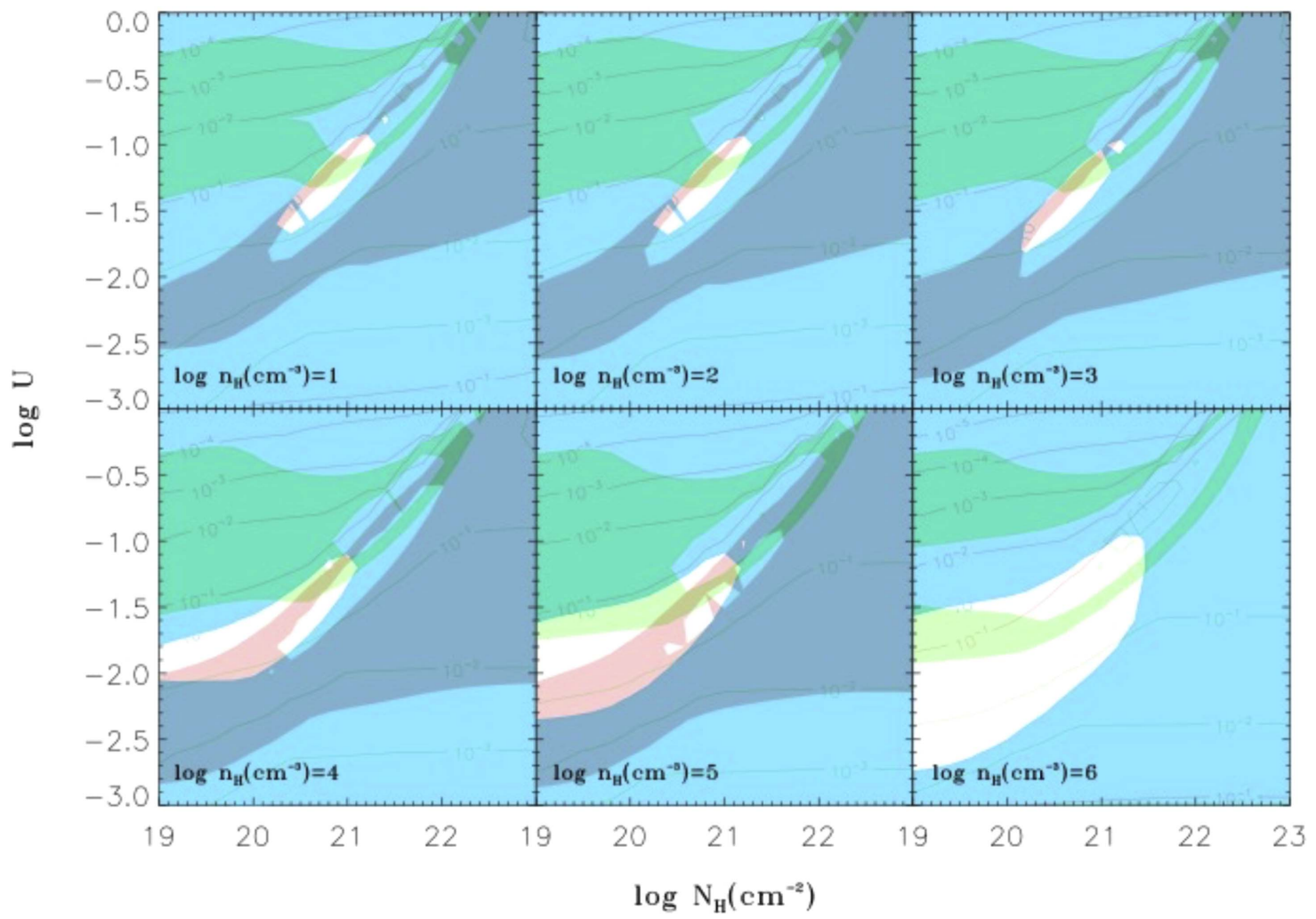

Figure 4. Upper limit of [C III]/Ly $\alpha$ overplotting the results of [O III] $\lambda 5007 / \mathrm{Ly} \alpha$ and $\mathrm{C}$ IV $/ \mathrm{Ly} \alpha$ in cyan. 


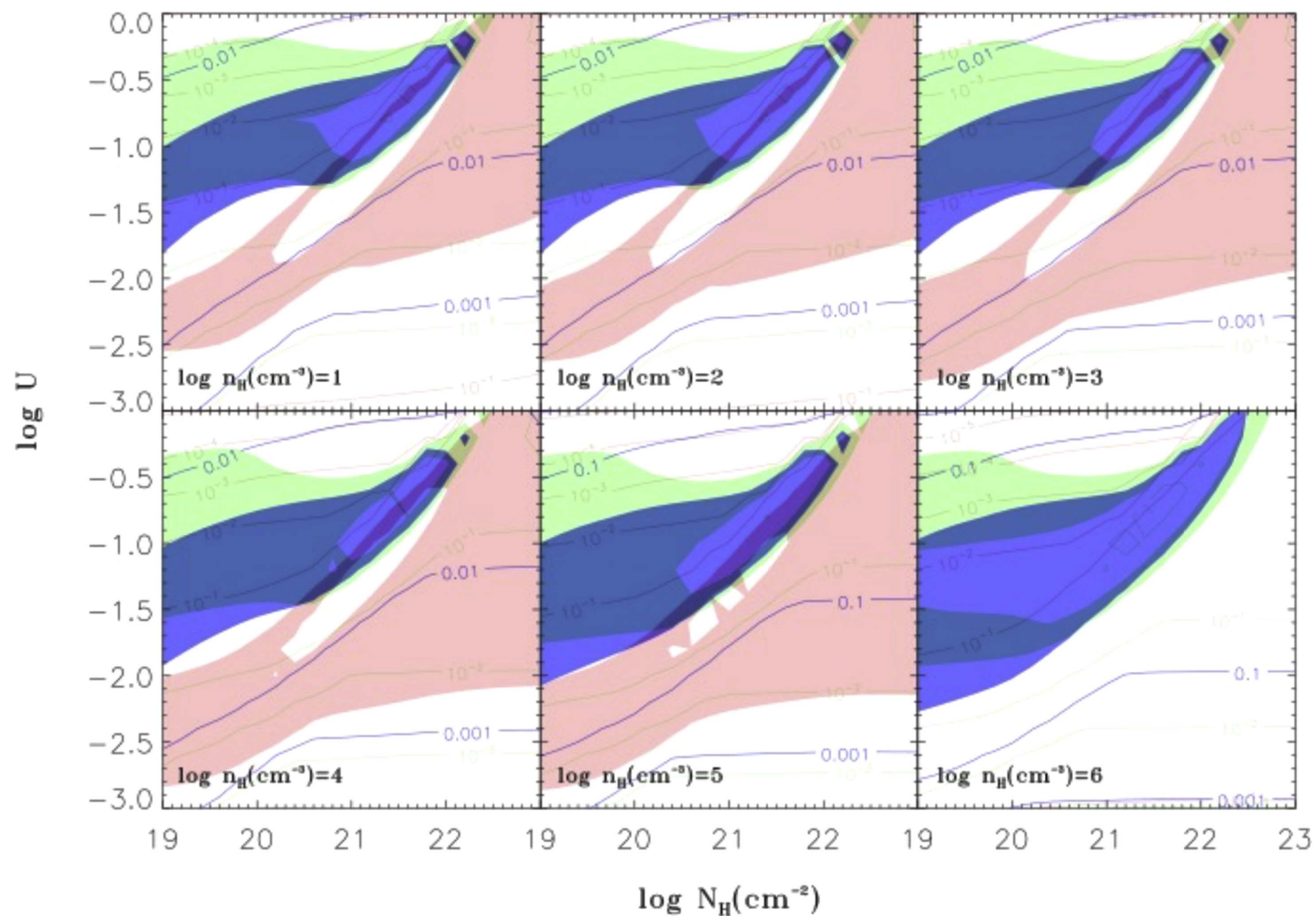

Figure 5. Flux ratios of Si IV $+[\mathrm{O}$ IV $] / \mathrm{Ly} \alpha$ overplotting the results of $[\mathrm{O}$ III $] \lambda 5007 / \mathrm{Ly} \alpha$ and $\mathrm{C}$ IV $/ \mathrm{Ly} \alpha$ in blue.

Section 4.2 of Dunn et al. 2010). We calculated a series of photoionization models with different ionization parameters, electron densities, and hydrogen column densities. The ranges of parameters are $-3 \leqslant \log _{10} U \leqslant 1,1 \leqslant \log _{10} n_{\mathrm{H}}\left(\mathrm{cm}^{-3}\right) \leqslant 6$, and $18 \leqslant \log _{10} N_{\mathrm{H}}\left(\mathrm{cm}^{-2}\right) \leqslant 24$, with a step of 0.2 dex.

We extract the simulated [O III] $\lambda 5007$, C IV, and Ly $\alpha$ fluxes from the Cloudy simulations and show the line ratios $[\mathrm{O}$ III $] \lambda 5007 / \mathrm{Ly} \alpha$ and $\mathrm{C}$ IV/Ly $\alpha$ by the red and green lines in Figure 3. The red and green areas show the observed $1 \sigma$ uncertainty ranges of $[\mathrm{O}$ III] $] \lambda 5007 / \mathrm{Ly} \alpha$ and $\mathrm{C}$ IV $/ \mathrm{Ly} \alpha$, so the overlapping region is the possible parameter space for the BBEL outflows of this object. Model calculations suggest that the gas clouds in two irregular regions with $\log _{10} N_{\mathrm{H}}\left(\mathrm{cm}^{-2}\right) \sim 21.0$ and $\log _{10} N_{\mathrm{H}}\left(\mathrm{cm}^{-2}\right) \sim 22.0$ can generate the observed line ratios of SDSS J0006 +1215 (Figure 3). Fortunately, the absence of [C III] gives the upper limit of $9.71 \times 10^{-17} \mathrm{erg} \mathrm{s}^{-1} \mathrm{~cm}^{-2}$, and the flux ratio of $[\mathrm{C} \mathrm{III]} / \mathrm{Ly} \alpha$ (cyan area in Figure 4) just covers the high column density region as the reliable parameter space. It is regrettable that the density cannot be more firmly confined. The Cloudy simulation gives a density range of $\log _{10} n_{\mathrm{H}}\left(\mathrm{cm}^{-3}\right) \leqslant 5.8$, which is an upper limit, as [O III $] \lambda \lambda 4959,5007$ also suggested. In Figure 5, the modeled and observed line ratios of $\mathrm{Si}$ IV $+[\mathrm{O}$ IV $]$ and $\mathrm{Ly} \alpha$ are shown in blue. It is clear that observed $\mathrm{SiIV}+[\mathrm{O}$ IV] $/ \mathrm{Ly} \alpha$ is overlapped with the $[\mathrm{O}$ III $] \lambda 5007 / \mathrm{Ly} \alpha$ and $\mathrm{CIV} / \mathrm{Ly} \alpha$ areas, but Si IV $+[\mathrm{O}$ IV $] / \mathrm{Ly} \alpha$ makes the possible parameter space of the outflowing gases narrower. In summary, the outflowing gases can survive in the parameter space with $\log _{10} n_{\mathrm{H}}\left(\mathrm{cm}^{-3}\right) \leqslant 5.8$, $\log _{10} U \sim-0.5$, and $\log _{10} N_{\mathrm{H}}\left(\mathrm{cm}^{-2}\right) \sim 22.0$. Actually, the reliable parameter-space region is essentially dynamic with the gas density.

\subsection{Kinetic Luminosity and Mass Flux of the Outflows}

Based on the physical properties determined by Cloudy, we estimate the distance $R$ of the outflows away from the central source. The ionization parameter $U$ depends on $R$ and the rate of hydrogen-ionizing photons emitted by the central source $Q$ and is given by

$$
U=\int_{\nu_{0}}^{\inf } \frac{L_{\nu}}{4 \pi R^{2} h \nu n_{\mathrm{H}} c} d \nu=\frac{Q}{4 \pi R^{2} n_{\mathrm{H}} c},
$$

in which $\nu_{0}$ is the frequency corresponding to the hydrogen edge, $n_{\mathrm{H}}$ is is the density of the outflows, and $c$ is the speed of light. To determine the hydrogen-ionizing rate $Q$, we scale the UV-soft SED to the extinction-corrected flux of SDSS J0006 +1215 at $5100 \AA$ (rest frame) and then integrate over the energy range $h \mu \geqslant 13.6 \mathrm{eV}$ of this scaled SED. This yields $Q=1.17 \times 10^{57}$ photons s$^{-1} . n_{\mathrm{H}}$ has been estimated as $n_{\mathrm{H}} \leqslant 10^{5.8} \mathrm{~cm}^{-3}$. Using this $Q$ value, together with the derived $n_{\mathrm{H}}$ and $U$, the lower limit of $R$ can be derived to be $41 \mathrm{pc}$. The radius of broad emission line regions, $R_{\mathrm{BLR}}$, can be estimated using the formula based on the luminosity at $5100 \AA$,

$$
R_{\mathrm{BELR}}=\alpha\left(\frac{L_{5100}}{10^{44} \mathrm{erg} \mathrm{s}^{-1}}\right)^{\beta} \mathrm{lt}-\text { days, }
$$

where the parameters $\alpha$ and $\beta$ are $30.2 \pm 1.4$ and $0.64 \pm 0.02$, respectively, given in Greene \& Ho (2005). Thus, the luminosity yields $R_{\mathrm{BLR}} \approx 1.3 \mathrm{pc}$. Meanwhile, the radius of the inner side of the dust torus, $R_{\text {Torus }}$, can also be estimated based on the thermal equilibrium of the inner side of the torus 
as

$$
R_{\text {Torus }}=\sqrt{\frac{L_{\mathrm{bol}}}{4 \pi \sigma T^{4}}},
$$

where $\sigma$ is the Stefan-Boltzmann constant, and $T(\sim 1500 \mathrm{~K})$ is the temperature of the inner side of the tours. Then, we get $R_{\text {Torus }} \approx 16$ pc. Formulae in Burtscher et al. (2013) and Kishimoto et al. (2011) gave the dust sublimation radii as 5.4 and $2.0 \mathrm{pc}$, respectively, smaller than the above estimation. In any case, the outflows are located externally of the torus. This result is consistent with the supposition of our spectral qualitative analysis, i.e., the dusty torus only obscures the continuum and the normal BELR rather than the BBEL outflows.

Assuming that the outflow materials can be described as a thin partially filled shell, the average mass-flow rate $(\dot{M})$ and kinetic luminosity $\left(\dot{E}_{k}\right)$ can be derived as follows (Borguet et al. 2012):

$$
\begin{gathered}
\dot{M}=4 \pi R \Omega \mu m_{p} N_{\mathrm{H}} v, \\
\dot{E}_{k}=2 \pi R \Omega \mu m_{p} N_{\mathrm{H}} v^{3},
\end{gathered}
$$

where $R$ is the distance of the outflows from the central source, $\Omega$ is the global covering fraction of the outflows, $\mu=1.4$ is the mean atomic mass per proton, $m_{p}$ is the mass of protons, $N_{\mathrm{H}}$ is the total hydrogen column density directly derived from the photoionization modeling of the outflow gases, and $v$ is the flux-weight-averaged velocity of the outflow gases. Here, $v$ is replaced by the blueshifted velocity of the BBEL peaks. We note that the BBELs originate from the gases outflowing along different directions with respect to the observer, and thus the observed outflow velocity of the BBEL is a sum of the projected velocities of the outflowing gases along different directions and should be a lower limit of the velocity of the outflow materials. Furthermore, we can estimate the global covering fraction for SDSS J0006 +1215 by comparing the measured $L_{[\mathrm{O} \text { III }] \lambda 5007}$ with the predicted one by the Cloudy model. In Cloudy modeling, the emergent values of $L_{[\mathrm{O} \text { III }] \lambda 5007}$ are output with the covering fraction being assumed to be 0.05 to 1 and are shown as a function of $n_{\mathrm{H}}(U)$ and $\Omega$ in Figure 6 . Meanwhile, we show the measured $\left.L_{[\mathrm{O}} \mathrm{II}\right] \lambda 5007$ with errors in gray. The comparison gives a global covering fraction for SDSS J0006+1215 of $\sim 6 \%-15 \%$. In the studies of BAL quasars, the fraction of BAL quasars is generally used as the global covering fraction of BAL outflow gases. The fraction is generally $10 \%-20 \%$ in optical-selected quasars (e.g., Trump et al. 2006; Gibson et al. 2010; Zhang et al. 2014) and 30\% (e.g., Maddox et al. 2008) or even 44\% (Dai et al. 2008) in NIR-selected quasars. We consider that BAL and BEL outflows may be different appearances viewed from different inclination angles but representing the same physical component, which is suggested by many works (e.g., Richards et al. 2011; Wang et al. 2011; Liu et al. 2016); the global covering fraction of BEL outflows will follow these values. The value we obtained in SDSS J0006 +1215 is slightly smaller than those estimated from optical-selected BAL quasars. Thus, for the upper limit of the density $n_{\mathrm{H}}=10^{5.8}\left(\mathrm{~cm}^{-3}\right)$ and the global covering fraction $\sim 10 \%$, the kinetic luminosity and mass-loss rate are calculated as $\dot{E}_{k}=1.74 \times 10^{43} \mathrm{erg} \mathrm{s}^{-1}$ and

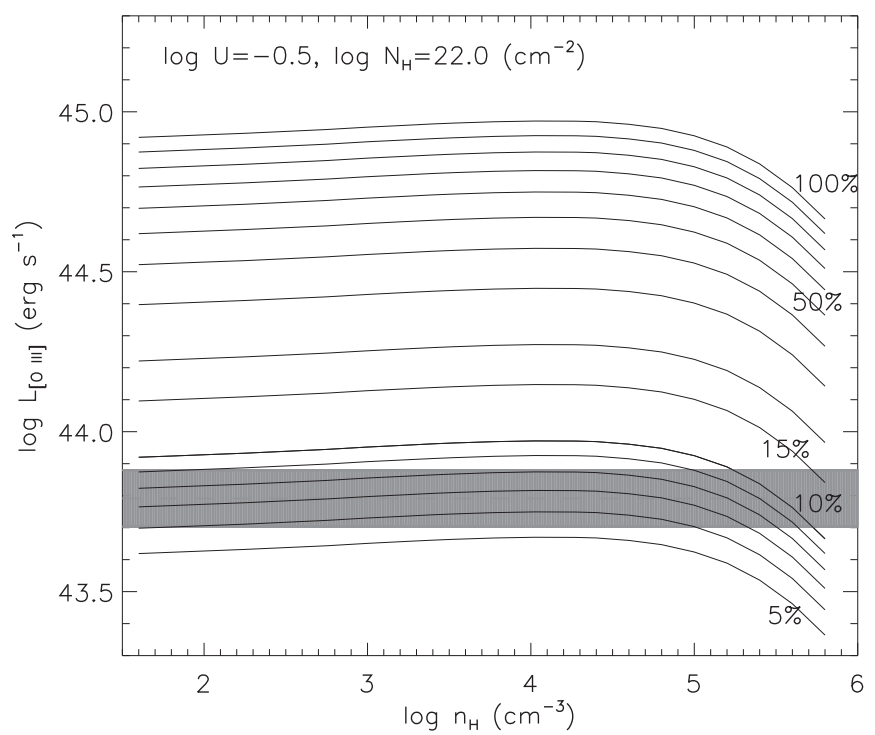

Figure 6. Luminosity of [O III] $\lambda 5007$ as a function of gas density and global covering fraction; the measured $L_{[\mathrm{O} \mathrm{II}] \lambda 5007}$ with $1 \sigma$ uncertainty is shown in gray.

$\dot{M}=12.23 M_{\odot} \mathrm{yr}^{-1}$, respectively, for $U=10^{-0.5}, N_{\mathrm{H}}=$ $10^{22.0} \mathrm{~cm}^{-3}$.

In general, the high-velocity outflows require kinetic luminosities to be of the order of a few percent of the Eddington luminosity (e.g., Scannapieco \& Oh 2004; Di Matteo et al. 2005; Hopkins \& Elvis 2010); however, in the case of SDSS J0006 +1215 , the reported lower limit on the kinetic luminosity $\left(\dot{E}_{k} \sim 10^{-5} L_{\text {Edd }}\right)$ is not of a sufficient value to efficiently drive AGN feedback. Note that this comparison is probably only a lower limit for the following reasons: (1) The weight-averaged velocity $v$ we use in Equation (5) is a sum of the projected velocities of the outflowing gases along different directions, the value of which is underestimated and should be a lower limit of the outflow velocity. (2) The distance of the outflows, $R$, is also a lower limit, and the outflow gases perhaps survive at greater distances from the central source.

\subsection{Origin of the Ly $\alpha$ Narrow Line}

As the only narrow emission line in SDSS J0006+1215, the Ly $\alpha$ narrow line presents strong emission. In theory Ly $\alpha$ photons scatter in neutral hydrogen until they either escape or are absorbed by dust grains. For SDSS J0006+1215, possible explanations are the NELR emission, the star formation in host galaxy, or the scattering of the obscured Ly $\alpha$ emission by the outflows. Before proceeding further, we first discuss whether the NELR and star-forming contribution are preferred in SDSS J0006 +1215 through other relevant narrow lines. If Ly $\alpha$ of SDSS J0006+1215 is the narrow emission line from the NELR, this situation can be confirmed through the presence of high-ionization lines, e.g., C IV in the rest-frame UV, or other lines such as He II that may also indicate AGN activity. The typical relative intensities of $\operatorname{Ly} \alpha$ and $\mathrm{C}$ IV narrow emission lines in Seyfert 2 spectra are 55 and 12, respectively (from Ferland \& Osterbrock 1986). Under this condition, the derived flux of the C IV narrow line is $\sim 4.54 \times 10^{-17} \mathrm{erg} \mathrm{s}^{-1} \mathrm{~cm}^{-2}$. Like the Ly $\alpha$ narrow line, we use one Gaussian with a width of $429 \mathrm{~km} \mathrm{~s}^{-1}$ to model the theoretical C IV narrow line, which is shown via the green line in the top panel of Figure 7. Apparently, the theoretical C IV narrow line would not appear 

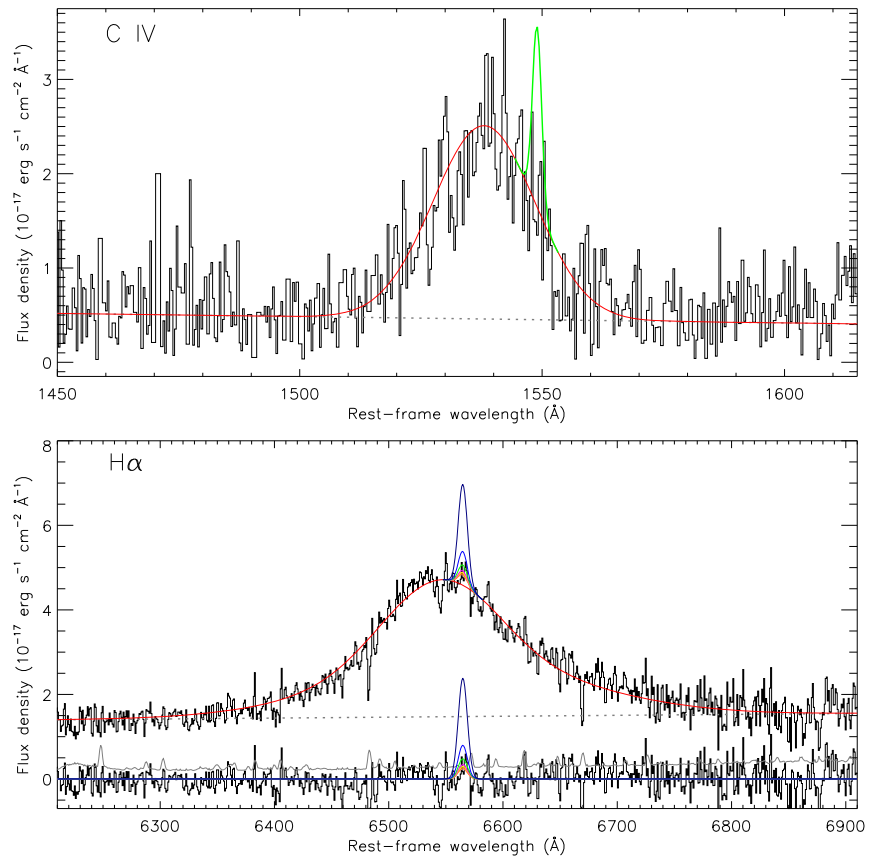

Figure 7. Top panel: theoretical C IV narrow line profile (green line) from the typical line ratio of Seyfert spectra. Bottom panel: theoretical $\mathrm{H} \alpha$ narrow line profiles (pink, orange, yellow magenta, green, blue, and navy lines) with $f_{\text {esc }}(\operatorname{Ly} \alpha)=90 \%, 80 \%, 70 \%, 50 \%, 30 \%$, and $10 \%$ in sequence, overplotted by the observed spectrum and the best-fitting profile (black and red line). The residual and observed error are also shown by black and gray lines for comparison.

in the spectrum of SDSS J0006+1215; otherwise, it should be detected at $\gtrsim 7 \sigma$.

With the assumption that the $\operatorname{Ly} \alpha$ emission is produced by photoionization by stars and the absence of extinction, one can estimate the $\mathrm{H} \alpha$ flux while assuming a case $\mathrm{B}$ recombination theory (Osterbrock 1989), $f_{\mathrm{esc}}(\operatorname{Ly} \alpha)=f(\operatorname{Ly} \alpha) /(8.7 \times$ $f(\mathrm{H} \alpha))$, where $f_{\mathrm{esc}}(\mathrm{Ly} \alpha)$ is the $\operatorname{Ly} \alpha$ escape fraction, $f(\operatorname{Ly} \alpha)$ is the observed Ly $\alpha$ flux, and $f(\mathrm{H} \alpha)$ is the $\mathrm{H} \alpha$ flux. When Ly $\alpha$ photons are $100 \%$ escaped, we obtain the flux and luminosity of $\mathrm{H} \alpha$ for the $\mathrm{H}$ II region, $f(\mathrm{H} \alpha)=(2.39 \pm 0.10) \times$ $10^{-17} \mathrm{erg} \mathrm{s}^{-1} \mathrm{~cm}^{-2}$ and $L_{\mathrm{H} \alpha}=(3.20 \pm 0.13) \times 10^{42} \mathrm{erg} \mathrm{s}^{-1}$. Following the related star formation rate $\operatorname{SFR}\left(M_{\odot} \mathrm{yr}^{-1}\right)=$ $7.9 \times 10^{-42} L_{\mathrm{H} \alpha}\left(\mathrm{erg} \mathrm{s}^{-1}\right)$ (Kennicutt 1998), SFR is estimated to be $\mathrm{SFR}=25.28 \pm 1.03 M_{\odot} \mathrm{yr}^{-1}$. When we use the profile of the $\mathrm{Ly} \alpha$ narrow line to model the theoretical $\mathrm{H} \alpha$ (pink line in the bottom panel of Figure 7), the height of this Gaussian profile is $0.26 \mathrm{erg} \mathrm{s}^{-1} \mathrm{~cm}^{-2} \AA^{-1}$, which is commensurate with the fluctuations of the NIR spectrum. Previous studies find that $f_{\text {esc }}(\operatorname{Ly} \alpha)$ is not constant and spans a wide range of values. At a similar redshift to SDSS J0006 +1215 , the average escape fraction from various methods is $5.3 \% \pm 3.8 \%$ by performing a blind narrowband survey in Ly $\alpha$ and $\mathrm{H} \alpha$ (Hayes et al. 2010), $29 \%$ at $1.9 \leqslant z \leqslant 3.5$ with the comparison between Ly $\alpha$ flux and dust-corrected UV continuum (Blanc et al. 2011), $>7 \%$ at $z=2.1$ and 3.1 with the comparison between Ly $\alpha$ flux and X-ray flux (Zheng et al. 2012), and $\sim 12 \%-30 \%$ at $z=2.2$ with the comparison between Ly $\alpha$ and $\mathrm{H} \alpha$ luminosity (Nakajima et al. 2012). In the bottom panel of Figure 7, we also show the theoretical $\mathrm{H} \alpha$ profiles with $f_{\mathrm{esc}}(\mathrm{Ly} \alpha)=90 \%, 80 \%, 70 \%, 50 \%, 30 \%$, and $10 \%$ in sequence. It becomes apparent that there is a threshold of almost no possibility of the existence of the $\mathrm{H} \alpha$ narrow line superposed on the $\mathrm{H} \alpha$ narrow line; however, it also cannot be ruled out by the low spectrum quality of the $\mathrm{H} \alpha$ regime of SDSS J0006+1215. Thus, the scattering of the obscured Ly $\alpha$ emission by the outflows is the most promising option.

Alternately, the NELR in AGNs is the largest spatial scale for the ionizing radiation from the central source. In the case of the NELR, the Ly $\alpha$ emission comes from a spatially extended region, so at least to some extent physical and kinematic distributions can be mapped out directly. In comparison to the NELR, the star-forming region in galaxies is generally of compact morphology and typically characterized by a small but resolved "core" with kiloparsec scales rather than the axisymmetric bi-conic ionized region. The high-resolution Ly $\alpha$ imaging will also clearly demonstrate the presence or location of the extent structures. For the possible participation of scattering, spectropolarimetric observations of the Ly $\alpha$ line will seek final clarification.

\section{Conclusion}

We performed a multiwavelength study of the continuum and emission lines of SDSS J0006+1215, a high-redshift quasar with striking "V"-shape SED turning at $i$ band and signatures of outflows in blueshifted emission lines. The multiwavelength SED analysis indicates that the continuum of SDSS J0006+1215 is heavily suppressed by dust reddening with $E(B-V)=0.7512 \pm 0.0049$, reducing the high luminosity of $L_{\mathrm{bol}}=2.63 \times 10^{47} \mathrm{erg} \mathrm{s}^{-1}$ to $M_{i}=24.17$, and the detected UV radiation comes from scattering. In the optical and NIR spectra, the normal BELR-originated emission lines and the continuum are obscured, and thus the emission-line outflows are significantly presented; even the emission profiles of $\mathrm{C} \mathrm{IV}, \mathrm{Ly} \alpha$, and $\mathrm{N} \mathrm{V}$ are totally dominated by the blueshifted components, suggestive of the AGN outflows. These lines can be used to constrain the physical properties of the outflowing gases by comparing the observed results with the model results of the photoionization synthesis code Cloudy. The physical parameters we determined for emission-line outflows are $U \sim 10^{-0.5}$ and $N_{\mathrm{H}} \sim 10^{22.0} \mathrm{~cm}^{-2}$, and the density is regrettably given an upper limit of $n_{\mathrm{H}} \sim 10^{5.8} \mathrm{~cm}^{-3}$. Using the luminosity of [O III] $\lambda 5007$ to obtain the global covering factor and the upper limit of the density, we estimate the lower limits on the distance of the outflow materials to the central source, the kinetic luminosity, and the mass-loss rate of the outflows, which are $R \sim 41 \mathrm{pc}, \quad \dot{E}_{k}=1.74 \times 10^{43} \mathrm{erg} \mathrm{s}^{-1}, \quad$ and $\dot{M}=12.23 M_{\odot} \mathrm{yr}^{-1}$, respectively. Under conditions of the NELR emission and star formation in the host galaxy, the strengths of the $\mathrm{CIV}$ and $\mathrm{H} \alpha$ narrow lines are investigated based on the relations with the Ly $\alpha$ narrow line. The nondetection of the $\mathrm{C}$ IV and $\mathrm{H} \alpha$ narrow lines suggests that the Ly $\alpha$ narrow line of SDSS J0006 +1215 is not likely to originate in the NELR or the star-forming region in the host galaxy. Perhaps the outflow gases, which have expanded to the inner region of the NELR, finally diffuse in the larger region and act as the scatterers of $\operatorname{Ly} \alpha$ photons.

This work is supported by the National Natural Science Foundation of China (NSFC-11573024, 11473025, 11421303) and the National Basic Research Program of China (the 973 Program 2013CB834905). T.J. is supported by the National Natural Science Foundation of China (NSFC-11503022) and the Natural Science Foundation of Shanghai (NO. 15ZR1444200). P.J. is supported by the National Natural 
Science Foundation of China (NSFC-11233002). We acknowledge the use of the Hale 200-inch Telescope at Palomar Observatory through the Telescope Access Program (TAP), as well as the archive data from the SDSS, UKIDSS, and WISE surveys. TAP is funded by the Strategic Priority Research Program, the Emergence of Cosmological Structures (XDB09000000), National Astronomical Observatories, Chinese Academy of Sciences, and the Special Fund for Astronomy from the Ministry of Finance. Observations obtained with the Hale Telescope at Palomar Observatory were obtained as part of an agreement between the National Astronomical Observatories, Chinese Academy of Sciences, and the California Institute of Technology. Funding for SDSSIII has been provided by the Alfred P. Sloan Foundation, the Participating Institutions, the National Science Foundation, and the U.S. Department of Energy Office of Science. The SDSSIII Web site is http://www.sdss3.org/.

\section{References}

Ahn, C. P., Alexandroff, R., Allende Prieto, C., et al. 2014, ApJS, 211, 17 Blanc, G. A., Adams, J. J., Gebhardt, K., et al. 2011, ApJ, 736, 31

Borguet, B. C. J., Edmonds, D., Arav, N., Dunn, J., \& Kriss, G. A. 2012, ApJ, 751, 107

Boroson, T. 2005, AJ, 130, 381

Burtscher, L., Meisenheimer, K., Tristram, K. R. W., et al. 2013, A\&A, 558, A149

Crenshaw, D. M. 1986, ApJS, 62, 821

Crenshaw, D. M., Schmitt, H. R., Kraemer, S. B., Mushotzky, R. F., \& Dunn, J. P. 2010, ApJ, 708, 419

Cushing, M. C., Vacca, W. D., \& Rayner, J. T. 2004, PASP, 116, 362

Dai, X., Shankar, F., \& Sivakoff, G. R. 2008, ApJ, 672, 108

Dawson, K. S., Schlegel, D. J., Ahn, C. P., et al. 2013, AJ, 145, 10

Di Matteo, T., Springel, V., \& Hernquist, L. 2005, Natur, 433, 604

Dong, X., Wang, T., Wang, J., et al. 2008, MNRAS, 383, 581

Dunn, J. P., Bautista, M., Arav, N., et al. 2010, ApJ, 709, 611

Ferland, G. J., Korista, K. T., Verner, D. A., et al. 1998, PASP, 110, 761

Ferland, G. J., \& Osterbrock, D. E. 1986, ApJ, 300, 658

Fitzpatrick, E. L., \& Massa, D. 1990, ApJS, 72, 163

Gaskell, C. M. 1982, ApJ, 263, 79

Gibson, R. R., Brandt, W. N., Gallagher, S. C., Hewett, P. C., \& Schneider, D. P. 2010, ApJ, 713, 220

Glikman, E., Helfand, D. J., \& White, R. L. 2006, ApJ, 640, 579

Gordon, K. D., Clayton, G. C., Misselt, K. A., Londolt, A. U., \& Wolff, M. J. 2003, ApJ, 594, 279

Granato, G. L., De Zotti, G., Silva, L., Bressan, A., \& Danese, L. 2004, ApJ, 600,580

Greene, J. E., \& Ho, L. C. 2005, ApJ, 630, 122

Hall, P. B. 2007, AJ, 133, 1271

Hall, P. B., Anderson, S. F., Strauss, M. A., et al. 2002, ApJS, 141, 267

Hamann, F., Korista, K. T., \& Morris, S. L. 1993, ApJ, 415, 541

Hamann, F., Zakamska, N. L., Ross, N., et al. 2017, MNRAS, 464, 3431

Hayes, M., Östlin, G., Schaerer, D., et al. 2010, Natur, 464, 562

Ho, L. C., \& Kim, M. 2015, ApJ, 809, 123

Hopkins, P. F., \& Elvis, M. 2010, MNRAS, 401, 7

Hopkins, P. F., Hernquist, L., Cox, T. J., \& Kereš, D. 2008, ApJS, 175, 356

Ji, T., Wang, T.-G., Zhou, H.-Y., \& Wang, H.-Y. 2012, RAA, 12, 369

Ji, T., Zhou, H., Jiang, P., et al. 2015, ApJ, 800, 56

Ji, T., Zhou, H.-Y., Wang, T.-G., \& Wang, H.-y. 2013, ChA\&A, 37, 17
Jiang, P., Ge, J., Zhou, H., Wang, J., \& Wang, T. 2011, ApJ, 732, 110 Kennicutt, R. C., Jr. 1998, ARA\&A, 36, 189

Kishimoto, M., Hönig, S. F., Antonucci, R., et al. 2011, A\&A, 536, A78

Komossa, S., Xu, D., Zhou, H., Storchi-Bergmann, T., \& Binette, L. 2008, ApJ, 680, 926

Lawrence, A., Warren, S. J., Almaini, O., et al. 2007, MNRAS, 379, 1599

Leighly, K. M. 2004, ApJ, 611, 125

Leighly, K. M., Cooper, E., Grupe, D., Terndrup, D. M., \& Komossa, S. 2015, ApJL, 809, L13

Leighly, K. M., \& Moore, J. R. 2004, ApJ, 611, 107

Li, Z., Zhou, H., Hao, L., et al. 2015, ApJ, 812, 99

Liu, W.-J., Zhou, H., Ji, T., et al. 2015, ApJS, 217, 11

Liu, W.-J., Zhou, H.-Y., Jiang, N., et al. 2016, ApJ, 822, 64

Maddox, N., Hewett, P. C., Warren, S. J., \& Croom, S. M. 2008, MNRAS, 386, 1605

Marziani, P., Sulentic, J. W., Dultzin-Hacyan, D., Calvani, M., \& Moles, M. 1996, ApJS, 104, 37

Marziani, P., Sulentic, J. W., Plauchu-Frayn, I., \& del Olmo, A. 2013, ApJ, 764,150

Nakajima, K., Ouchi, M., Shimasaku, K., et al. 2012, ApJ, 745, 12

Ogle, P. M., Cohen, M. H., Miller, J. S., et al. 1999, ApJS, 125, 1

Osterbrock, D. E. 1989, Astrophysics of Gaseous Nebulae and Active Galactic Nuclei (Mill Valley, CA: University Science Books)

Palanque-Delabrouille, N., Magneville, C., Yèche, C., et al. 2016, A\&A, 587, A41

Rafiee, A., Pirkola, P., Hall, P. B., et al. 2016, MNRAS, 459, 2472

Reichard, T. A., Richards, G. T., Hall, P. B., et al. 2003, AJ, 126, 2594

Richards, G. T., Kruczek, N. E., Gallagher, S. C., et al. 2011, AJ, 141, 167

Richards, G. T., Vanden Berk, D. E., Reichard, T. A., et al. 2002, AJ, 124, 1

Ross, N. P., Hamann, F., Zakamska, N. L., et al. 2015, MNRAS, 453, 3932

Ross, N. P., McGreer, I. D., White, M., et al. 2013, ApJ, 773, 14

Runnoe, J. C., Brotherton, M. S., \& Shang, Z. 2012, MNRAS, 422, 478

Scannapieco, E., \& Oh, S. P. 2004, ApJ, 608, 62

Scarpa, R., \& Falomo, R. 1997, A\&A, 325, 109

Schlegel, D. J., Finkbeiner, D. P., \& Davis, M. 1998, ApJ, 500, 525

Shi, X., Zhou, H., Shu, X., et al. 2016, ApJ, 819, 99

Smee, S. A., Gunn, J. E., Uomoto, A., et al. 2013, AJ, 146, 32

Snedden, S. A., \& Gaskell, C. M. 2007, ApJ, 669, 126

Trump, J. R., Hall, P. B., Reichard, T. A., et al. 2006, ApJS, 165, 1

Vacca, W. D., Cushing, M. C., \& Rayner, J. T. 2003, PASP, 115, 389

Vanden Berk, D. E., Richards, G. T., Bauer, A., et al. 2001, AJ, 122, 549

Véron-Cetty, M.-P., Joly, M., \& Véron, P. 2004, A\&A, 417, 515

Wang, H., Wang, T., Zhou, H., et al. 2011, ApJ, 738, 85

Wang, H., Xing, F., Zhang, K., et al. 2013, ApJL, 776, L15

Wang, H.-Y., Wang, T.-G., \& Wang, J.-X. 2007, ApJS, 168, 195

Weymann, R. J., Morris, S. L., Foltz, C. B., \& Hewett, P. C. 1991, ApJ, 373,23

Wilkes, B. J. 1984, MNRAS, 207, 73

Williams, R. J., Maiolino, R., Krongold, Y., et al. 2016, arXiv:1605.08046

Wilson, J. C., Henderson, C. P., Herter, T. L., et al. 2004, Proc. SPIE, 5492,1295

Wright, E. L., Eisenhardt, P. R. M., Mainzer, A. K., et al. 2010, AJ, 140,1868

York, D. G., Adelman, J., Anderson, J. E., Jr., et al. 2000, AJ, 120, 1579

Zakamska, N. L., Hamann, F., Pâris, I., et al. 2016, MNRAS, 459, 3144

Zamanov, R., Marziani, P., Sulentic, J. W., et al. 2002, ApJL, 576, L9

Zhang, K., Dong, X.-B., Wang, T.-G., \& Gaskell, C. M. 2011, ApJ, 737, 71

Zhang, S., Ge, J., Jiang, P., et al. 2015a, ApJ, 802, 92

Zhang, S., Wang, H., Wang, T., et al. 2014, ApJ, 786, 42

Zhang, S., Wang, T.-G., Wang, H., et al. 2010, ApJ, 714, 367

Zhang, S., Zhou, H., Shi, X., et al. 2015b, ApJ, 815, 113

Zhang, S., Zhou, H., Wang, T., et al. 2015c, ApJ, 803, 58

Zheng, Z.-Y., Malhotra, S., Wang, J.-X., et al. 2012, ApJ, 746, 28 